\title{
AWAKE FIBEROPTIC INTUBATION THROUGH THE I-GEL SUPRAGLOTTIC DEVICE IN CERVICAL SPINE INJURY
}

\author{
J.Arévalo MD. PhD. S. Gallardo, J.J.Arcas, V. López MD. PhD, L. E. Muñoz MD. PhD, M.Vázquez \\ University Hospital Fundacion Jimenez Diaz, Dept of Anaesthesiology, Madrid, Spain
}

\section{Background:}

- Direct laryngoscopy for orotracheal intubation may be difficult in patients with cervical spine injury due to limitation in neck mobility'. These patients may benefit from intubation techniques with no movements in cervical anatomy ${ }^{2}$. Fiberoptic intubation through the l-gel device can be carried out without the need of neck flexo-extension and rotation movements.

\section{Case Report:}

- A 32 year old man who suffered cervical vertebral fractures in C3 and C4 due to a car accident was taken into the theater under spontaneous ventilation and conscious.

- He also presented other difficult airway predictors, such as beard, retrognatia and limited mouth opening $(2,5 \mathrm{~cm})$.

- He refused awake intubation. So we decided to administer oropharyngeal local anesthesia and then induce sedation in order to insert the l-gel under spontaneous breathing.

- After confirming proper ventilation through the l-gel with capnography curve, the fiberscope was inserted with a reinforced endotracheal tube (ETT) over it.

- Once the glottic structures were observed, general anesthesia with neuromuscular relaxants was induced thus preventing coughing and neck movements. The fiberscope was then passed through the vocal cords until the carina was seen and finally the ETT was railroaded over the fiberscope inside the airway.

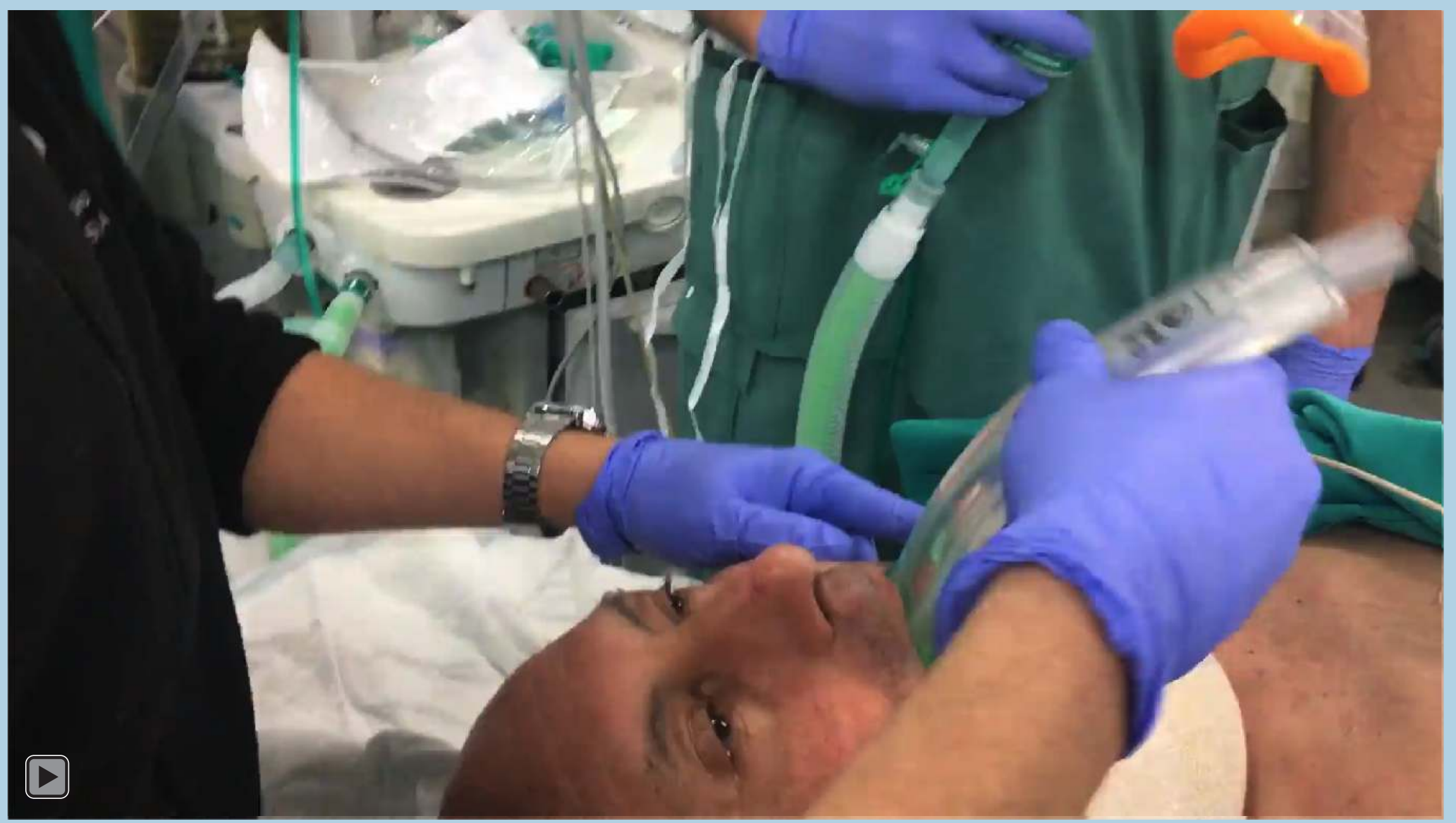

\section{Discussion:}

- Cervical vertebral fractures are one of the most dangerous clinical situations that anesthesiologists could face.

- Once cervical stabilization is achieved, endotracheal intubation should be performed without any neck movement not to damage the spinal cord. By using the l-gel to achieve a patent airway, we prevent the patient from suffering neck mobility.

- The l-gel lies above the glottic structures facilitating the passage of the fiberscope through the vocal cords as few maneuverability is needed.

\section{Conclussion:}

- Both Airtraq ${ }^{\circledR}$ and fiberoptic guided intubation through the l-gel $®$ are safe and effective techniques to achieve intubation in patients with cervical spine stabilization and reduction of neck mobility.

- Fiberoptic intubation through the I-gel $@$ seems to be easier to perform than Airtraq intubation in simulated cervical stabilization

\section{Learning points:}

- Neck movements should be avoided to achieve endotracheal intubation in cases of cervical vertebral fractures.

- The l-gel can be inserted in spontaneous ventilation in cases of cervical spine injury.

- Fiberscope intubation through the l-gel device can be performed without the need for flexo-extension neck movements.

\section{References:}

I.Naola Austin et al.Airway management in cervical spine injury. Int J Crit Illn Inj Sci. 2014 Jan-Mar; 4(I): 50-56.

2.Waltl B et al.Tracheal intubation and cervical spine excursion: direct laryngoscopy vs. intubating laryngeal mask.Anaesthesia. 200I Mar:56(3):22I-6 